\title{
Two- and three-dimensional magnetic ordering in the bilayer manganite $\mathrm{Ca}_{2.5} \mathrm{Sr}_{0.5} \mathrm{GaMn}_{2} \mathrm{O}_{8}$
}

\author{
S. M. Yusuf, ${ }^{1,2, *}$ J. M. De Teresa, ${ }^{1}$ P. A. Algarabel, ${ }^{1}$ M. D. Mukadam, ${ }^{2}$ I. Mirebeau, ${ }^{3}$ J.-M. Mignot,${ }^{3}$ \\ C. Marquina, ${ }^{1}$ and M. R. Ibarra ${ }^{1,4}$ \\ ${ }^{1}$ Instituto de Ciencia de Materiales de Aragón, Universidad de Zaragoza-CSIC, Zaragoza 50009, Spain \\ ${ }^{2}$ Solid State Physics Division, Bhabha Atomic Research Centre, Mumbai 400 085, India \\ ${ }^{3}$ Laboratoire Léon Brillouin, CEA-CNRS, CEA/Saclay, 91191 Gif sur Yvette, France \\ ${ }^{4}$ Instituto de Nanociencia de Aragón, Universidad de Zaragoza, Zaragoza 50009, Spain \\ (Received 18 September 2006; revised manuscript received 26 September 2006; published 6 November 2006)
}

Neutron diffraction study as a function of temperature on the bilayered manganite $\mathrm{Ca}_{2.5} \mathrm{Sr}_{0.5} \mathrm{GaMn}_{2} \mathrm{O}_{8}$ has revealed antiferromagnetic correlations between the [010] oriented $\mathrm{Mn}$ spins in the $a-c$ plane. Threedimensional (3D) short-range and two-dimensional (2D) long-range order over the temperature ranges 180-170 K and 165-160 K, respectively, have been observed. Below $\sim 150 \mathrm{~K}$, a pure 3D long-range ordering has been observed. Finally a phase diagram is proposed. A qualitative connection is made between the $2 \mathrm{D}$ and 3D magnetic ordering to the transport and magnetotransport properties reported for this system.

DOI: 10.1103/PhysRevB.74.184409

PACS number(s): 75.30.-m, 75.40.Cx, 75.47.Lx, 75.50.Ee

\section{INTRODUCTION}

The reduction of dimensionality for magnetic systems often results in new and exciting magnetic properties. The physics of low-dimensional magnetism and its crossover to higher dimensions are some of the outstanding open issues in condensed matter physics. As per the theorem of Mermin and Wagner, ${ }^{1}$ an infinite $d$ dimensional lattice of localized spins cannot have long-range order (LRO) at any finite temperature for $d<3$ if the effective exchange interactions among spins are isotropic in spin space and of finite range. These conditions are precisely satisfied by the Heisenberg theory of localized spins. The theorem has been recently generalized by Bruno $^{2}$ by including long-range exchange interactions. When anisotropy (such as single ion anisotropy, nonisotropic exchange constants, and dipole-dipole interactions) and/or infinite-range interactions (e.g., the RKKY interactions or the classical dipolar interactions) are considered, magnetic LRO at finite $T$ is possible even in lowdimensional $(d<3)$ systems. ${ }^{3-5}$ There has been a considerable amount of research on two-dimensional (2D) and three-dimensional (3D) magnetic ordering and their relationship with other important physical properties in highly anisotropic magnetic compounds, for example in parent systems of high- $T_{C}$ superconductors $\left(\mathrm{La}_{2} \mathrm{CuO}_{4}\right.$ and $\left.\mathrm{YBa}_{2} \mathrm{Cu}_{2} \mathrm{O}_{6}\right),{ }^{6}$ layered borocarbides $R \mathrm{~B}_{2} \mathrm{C}$ ( $R=\mathrm{Dy}, \mathrm{Ho}$, and Er), ${ }^{7}$ and layered manganites $\mathrm{La}_{3-x} \mathrm{Sr}_{x} \mathrm{Mn}_{2} \mathrm{O}_{7} .{ }^{8} \mathrm{La}_{2} \mathrm{CuO}_{4}$ and $\mathrm{YBa}_{2} \mathrm{Cu}_{2} \mathrm{O}_{6}$ compounds show fairly high Neel temperature $T_{\mathrm{N}}$ and strongly anisotropic antiferromagnetic correlations, indicating that the effective exchange coupling $J_{\|}$within the $\mathrm{CuO}_{2}$ planes is unusually large $(\sim 0.1 \mathrm{eV})$ while the anisotropy ratio $\varepsilon\left(=J_{\perp} / J_{\|}\right)$is quite small $(\ll 1){ }^{6}$ Here the ratio between the out-of-plane and in-plane exchange interactions $\varepsilon$ controls the dimensional crossover of magnetic ordering from 3D $(\varepsilon \sim 1)$ to $2 \mathrm{D}(\varepsilon \rightarrow 0)$ as well as anisotropic transport, magnetotransport and other physical properties. The high-temperature superconducting oxides involving rare earth magnetism ${ }^{9-11}$ show 2D-3D crossover behavior at quite low temperature $(\sim 1 \mathrm{~K})$. This suggests that the exchange interactions are small in the crystallographic $a b$ plane and very small in the $c$ direction, leaving the classical dipole interactions to dominate. In the classical 2D systems, such as $\mathrm{K}_{2} \mathrm{CoF}_{4}$ and $\mathrm{K}_{2} \mathrm{NiF}_{4}\left(T_{\mathrm{N}}=97.23 \mathrm{~K}\right.$ for $\left.\mathrm{K}_{2} \mathrm{NiF}_{4}\right)$, the $2 \mathrm{D}$ and $3 \mathrm{D}$ magnetic orderings occur at essentially the same temperature. ${ }^{12,13}$

Recently it has been shown by neutron diffraction ${ }^{14}$ that $\mathrm{Ca}_{2.5} \mathrm{Sr}_{0.5} \mathrm{GaMn}_{2} \mathrm{O}_{8}$ has a perovskite structure in which perovskite bilayers consisting of two $\mathrm{MnO}_{6}$ octahedra layers are isolated from each other by a single layer of $\mathrm{GaO}_{4}$ tetrahedra. At $5 \mathrm{~K}$, the compound is reported to be antiferromagnetic $\left(T_{\mathrm{N}} \sim 150 \mathrm{~K}\right)$ with an ordered magnetic moment of 3.09(1) $\mu_{\mathrm{B}}$ per Mn cation (aligned along [010]), which is consistent with the presence of a 1:1 ratio of $\mathrm{Mn}^{3+}$ and $\mathrm{Mn}^{4+}$ ions on the octahedral sites. ${ }^{14}$ However, a short-range magnetic ordering between $\sim 150 \mathrm{~K}$ and $200 \mathrm{~K}$ was found in susceptibility ${ }^{14}$ as well as in a later diffraction study. ${ }^{15}$

In this paper we study the magnetic ordering in this $\mathrm{Mn}$ based naturally bilayered oxide system $\mathrm{Ca}_{2.5} \mathrm{Sr}_{0.5} \mathrm{GaMn}_{2} \mathrm{O}_{8}$. We demonstrate that crossovers from short-range $3 \mathrm{D}$ to longrange $2 \mathrm{D}$ then to long-range $3 \mathrm{D}$ occur over a significantly wide temperature range. Moreover, this compound has a high magnetic ordering temperature (3D long-range antiferromagnetic Néel temperature $\left.T_{\mathrm{N}} \sim 150 \mathrm{~K}\right)$ and superexchange interactions are responsible for magnetic ordering. Furthermore, in this manganite system, resistivity drops by an order of magnitude on cooling through $T_{\mathrm{N}}$ and a large negative magnetoresistance MR $(\sim 50 \%)$ is observed around the Néel temperature. ${ }^{14}$ This class of manganite system is, therefore, highly relevant to the ongoing search for new oxide materials in the field of spin electronics. The bilayered brownmillerite material $\mathrm{Ca}_{2.5} \mathrm{Sr}_{0.5} \mathrm{GaMn}_{2} \mathrm{O}_{8}$ studied here can be at some context comparable to the two important members of the $A_{\mathrm{n}+1} \mathrm{Mn}_{\mathrm{n}} \mathrm{O}_{3 \mathrm{n}+1}$ Ruddlesden-Popper (RP) series, namely the $n=\infty$ perovskites, for example $\mathrm{La}_{1-x} A_{x} \mathrm{MnO}_{3}$ with $A$ : Ca, $\mathrm{Sr}$, $\mathrm{Pb}$, etc. and their derivatives ${ }^{16}$ and the $n=2$ phases, for example $\mathrm{La}_{3-x} \mathrm{Sr}_{x} \mathrm{Mn}_{2} \mathrm{O}_{7}$ (Ref. 8) which have drawn great attention for their colossal magnetoresistance (CMR) behavior and interesting intertwined magnetic, transport and structural properties. 


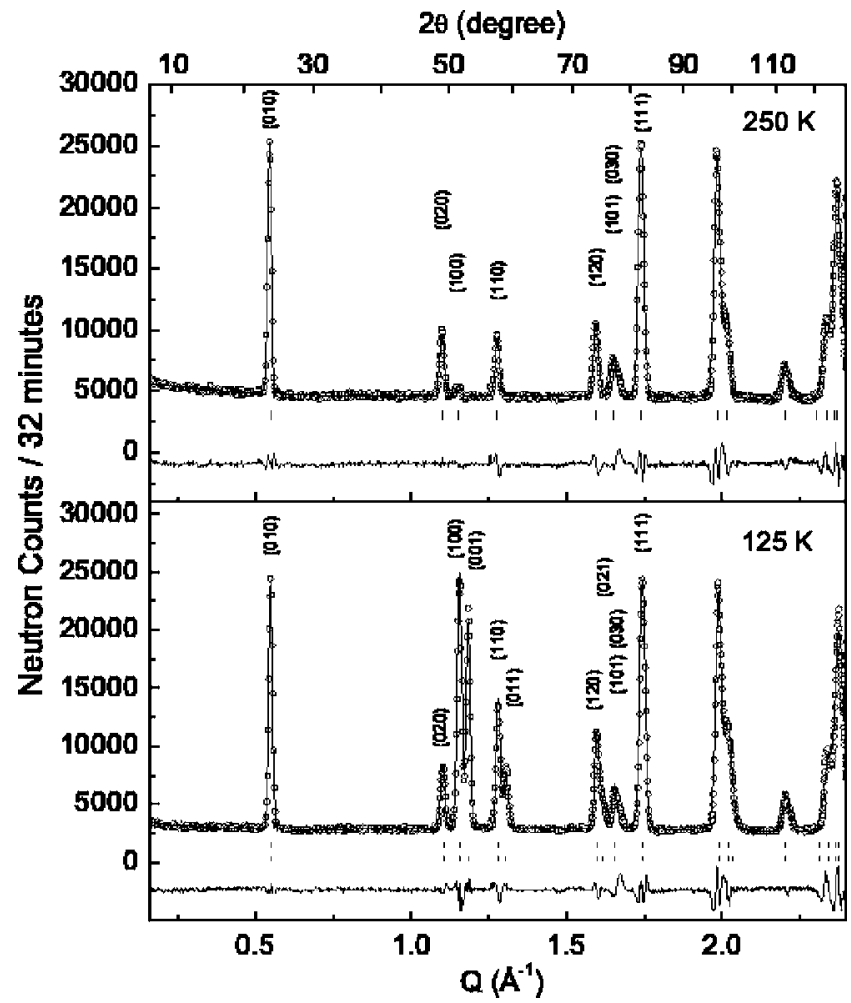

FIG. 1. Observed (open circles) and Rietveld refined (solid lines) neutron diffraction patterns at $250 \mathrm{~K}$ (top layer) and $125 \mathrm{~K}$ (bottom layer). Differences between observed and calculated patterns are also shown by solid lines at the bottom of each panel. The difference patterns are shifted downward for the sake of clarity. The vertical bars indicate the position of allowed Bragg peaks. At $125 \mathrm{~K}$, the position of magnetic Bragg peaks is also shown. The $(h \mathrm{kl})$ values are also listed for the lower angle peaks.

\section{EXPERIMENTAL}

The polycrystalline sample was prepared by the solid state reaction method as described elsewhere. ${ }^{14}$ Unpolarized neutron diffraction measurements were carried out in the temperature range $250-125 \mathrm{~K}$ with a wavelength $\lambda=4.741 \AA$ covering a scattering vector length $[Q=(4 \pi / \lambda) \sin \theta]$ of $\sim 0.120-2.40 \AA^{-1}$ using the G6-1 powder neutron diffractometer at LLB, Saclay. For these measurements, sample was placed in a vanadium can and a commercial liquid helium cryostat was used for temperature variation.

\section{RESULTS AND DISCUSSION}

Figure 1 shows the observed and Rietveld refined neutron powder diffraction patterns at 250 and $125 \mathrm{~K}$. Rietveld refinement was performed with the FULLPROF $\operatorname{program}^{17}$ in the WINPLOTR suite of programs. Refinement shows the singlephase nature of the sample with orthorhombic crystal structure (space group $P \mathrm{~cm} 22_{1}$ ). The diffraction pattern at $250 \mathrm{~K}$ could be fitted with only nuclear intensities confirming the paramagnetic nature of the sample at this temperature. The resulting lattice constants are $a=5.4506(7), b=11.408(2)$, and $c=5.3221(9) \AA$. The Rietveld refinement of the pattern at $125 \mathrm{~K}$ confirms the $3 \mathrm{D}$ long-range antiferromagnetic

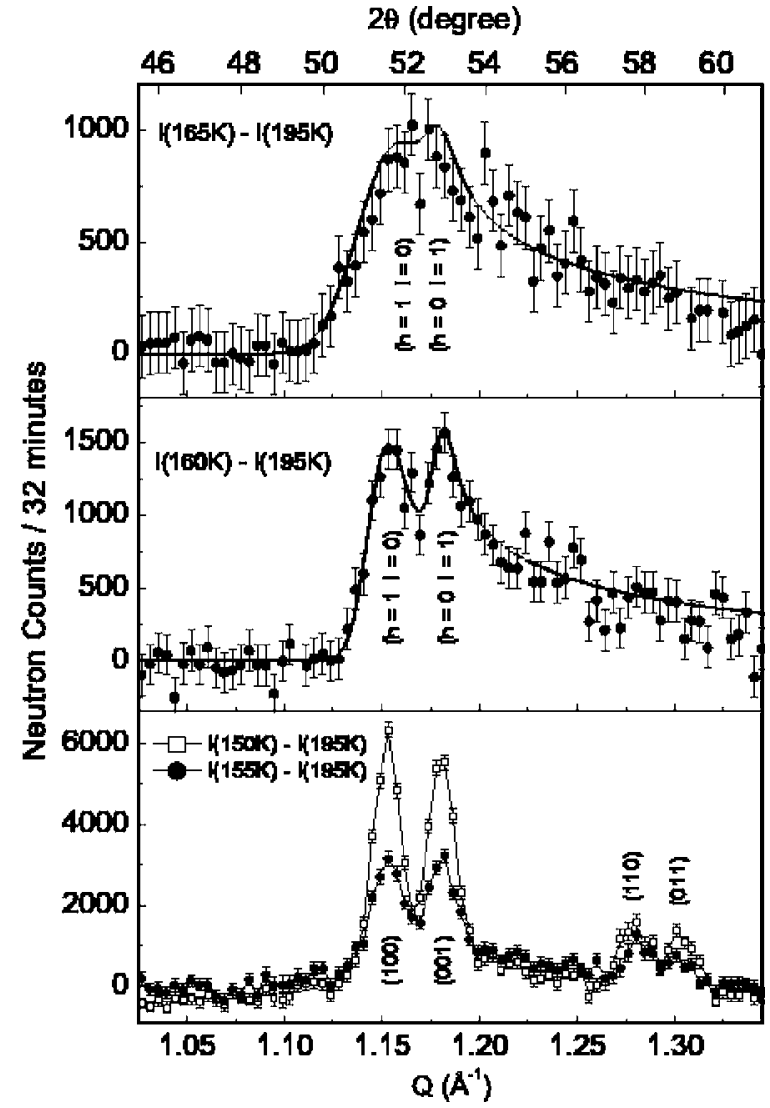

FIG. 2. Magnetic diffraction patterns at 165, 160, 155, and $150 \mathrm{~K}$. Nuclear background at $195 \mathrm{~K}$ has been subtracted out. The solid curves in the upper two panels are calculated profiles using the 2D theoretical Warren function. Whereas, in the bottom panel they are just guide to the eye.

structure in which Mn spins are ordered $\left[\mu_{\mathrm{Mn}}=2.31(5) \mu_{\mathrm{B}}\right]$ along the long $b$ axis, which mimics an Ising-like spin structure. ${ }^{18}$ The obtained spin arrangement is found to be consistent with the earlier report ${ }^{14}$ i.e., in the same $a-c$ planar sheet, each Mn spin is coupled antiferromagnetically to its four nearest neighbor Mn spins, and ferromagnetically to the single nearest-neighbor Mn spin in the other sheet of the same bilayer. A ferromagnetic inter-bilayer coupling is found. In Figs. 2 and 3 we show the magnetic diffraction patterns at $150,155,160,165,170,175$, and $180 \mathrm{~K}$ that were obtained after subtracting the higher temperature (195 K) diffraction data consisting of only nuclear Bragg peaks. At the higher temperatures $(\sim 180-170 \mathrm{~K})$ a broad peak centered at $2 \theta \approx 52 \operatorname{deg}\left(Q \sim 1.16 \AA^{-1}\right)$ is found (Fig. 3 ). With decreasing temperature (over $165-160 \mathrm{~K}$ ) this broad peak gradually transforms to two saw-tooth type asymmetric Bragg peaks centered on $2 \theta \approx 51.3$ and $52.7 \mathrm{deg}$ (Fig. 2). However, at $T<160 \mathrm{~K}$ (over $155-150 \mathrm{~K}$ ), the sawtooth type asymmetric peaks gradually disappear and symmetric Bragg peaks appear at the same scattering angles (Fig. 2 ). The temperature dependence of these difference patterns confirms that these broad/asymmetric/symmetric peaks are due to magnetic scattering. The absence of any temperature dependent intensity (apart from a weak Debye Waller factor) of the $(0 k 0)$ nuclear (fundamental) Bragg peaks, such as, 


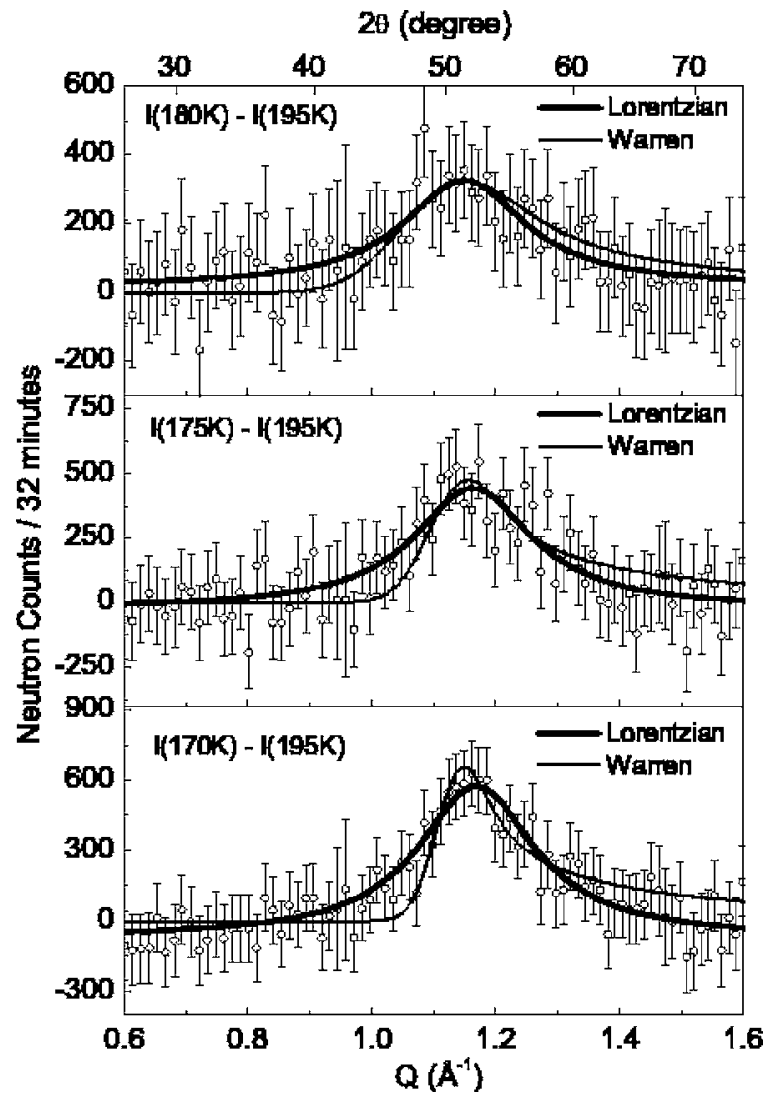

FIG. 3. Diffraction patterns at 180,175 , and $170 \mathrm{~K}$ after subtracting the observed diffraction pattern at $195 \mathrm{~K}$. The thin and thick solid curves are calculated profiles using the $2 \mathrm{D}$ theoretical Warren function and 3D Lorentzian function, respectively.

(010) and (020) confirms that the ordered Mn moments are always aligned along the [010] direction. Over the range $155-150 \mathrm{~K}$, the symmetric Bragg peaks that develop centered at $2 \theta=51.54(1), 52.91(1), 57.67(5)$, and 58.95(5) deg, have been indexed as (100), (001), (110), and (011), respectively, signaling that a $3 \mathrm{D}$ long-range antiferromagnetic correlation has built up. However, at $155 \mathrm{~K}$ some degree of the asymmetric nature of these Bragg peaks is still evident. At further lower temperatures $(T<150 \mathrm{~K})$ these symmetric Bragg peaks fully develop (see Fig. 1) confirming the formation of a pure $3 \mathrm{D}$ long-range antiferromagnetic correlation as reported by Battle et al. ${ }^{14}$

The saw-tooth type asymmetric diffraction profiles observed at 165 and $160 \mathrm{~K}$ [Fig. 2] are the typical signature of a 2 D LRO. ${ }^{9,10,19}$ For the present system where there is no effective magnetic exchange coupling along the crystallographic $b$ direction (or much weaker than the in-plane exchange coupling $J_{a c}$ ), the lattice for magnetic scattering is confined in the crystallographic $a-c$-plane. In this case, the momentum transfer is restricted to only two dimensions. As a result, there is no magnetic Bragg point (expected for a 3D magnetic ordering of a single crystal) in the reciprocal space, ${ }^{9,10}$ but rather Bragg lines or Bragg rods extending along the third ( $b$ axis) dimension. For a powder sample, an averaging of this scattering over all possible orientations of reciprocal space is necessary. Since the momentum transfer is only defined in two dimensions, there is a minimum transfer of $Q\left[Q_{\min }=(4 \pi / \lambda) \sin \theta_{2 \mathrm{DB}}\right]$, but no maximum. This causes the asymmetric Bragg peak profile with a step increase at $Q_{\min }$ and an intensity decreasing slowly at large scattering angles in contrast with the sharp symmetric peaks that occur in the 3D case. The degree of asymmetry of the powder pattern depends on the 2D correlation length. Following Warren, ${ }^{19}$ the diffracted intensity for the $(h l)$ 2D Bragg reflection is given by the following expression:

$$
\begin{aligned}
I_{h l}(2 \theta)= & C\left[\xi_{2 \mathrm{D}} /(\lambda \sqrt{\pi})\right]^{1 / 2} j_{h l}\left|F_{h l}\right|^{2} \\
& \times\left(1+\cos ^{2} 2 \theta\right) F(a) / 2(\sin \theta)^{3 / 2},
\end{aligned}
$$

where $C$ is a scale factor, $\xi_{2 \mathrm{D}}$ is the $2 \mathrm{D}$ spin-spin correlation length within the 2D layer, $\lambda$ is the neutron wavelength, $j_{h l}$ is the multiplicity of the $2 \mathrm{D}$ reflection $(h l)$ with magnetic structure factor $F_{h l}$, and $2 \theta$ is the scattering angle. The function $F(a)$ is given by $F(a)=\int_{0}^{\infty} \exp \left[-\left(x^{2}-a\right)^{2}\right] d x$, where $a$ $=\left(2 \xi_{2 \mathrm{D}} \sqrt{\pi} / \lambda\right)\left(\sin \theta-\sin \theta_{2 \mathrm{DB}}\right)$. The expression (1) has been calculated numerically to obtain the best possible fit of the experimental data in the $165-160 \mathrm{~K}$ temperature range (Fig. 2 ). We had to sum up contribution from two $2 \mathrm{D}$ Bragg peaks at $2 \theta=51.30$ and $52.70 \mathrm{deg}$. The mean $2 \mathrm{D}$ correlation length $\xi_{2 \mathrm{D}}$ was found to be 325 and $550 \AA$ at 165 and $160 \mathrm{~K}$, respectively. A trend of lower $\xi_{2 \mathrm{D}}$ for the lower angle peak $\left(2 \theta=51.30^{\circ}\right)$ is evident in our analysis. However, a systematic neutron diffraction study on a single crystal sample would be required to gain reliable quantitative information on such anisotropic behavior of the in-plane magnetic correlation. At $155 \mathrm{~K}$, a proper model representation of the observed profile is difficult due to the appearance of $3 \mathrm{D}$ LR correlation. At $T<150 \mathrm{~K}\left(T_{\mathrm{N}-3 \mathrm{D}}\right)$ the 3D Bragg peak widths are limited by the instrumental $Q$ resolution. For the present system at lower temperatures when there is 3D LRO, a ferromagnetic coupling between the neighboring two Mn layers (intrabilayer) is found. For 2D ordering from a such coupledintrabilayer system, one expects a sinusoidal oscillation of the saw-tooth type profile of the scattered intensity. ${ }^{10} \mathrm{How}-$ ever, a single crystal neutron diffraction study would be required to observe any such modulation in the diffraction profiles.

The observed diffraction profiles at 180,175 , and $170 \mathrm{~K}$ (shown in Fig. 3) have been analyzed using the Warren function [Eq. (1)] as well as the Lorentzian expression $I(Q)$ $=A /\left[\left|Q-Q_{c}\right|^{2}+\kappa^{2}\right]$. Here $\kappa$ is the inverse of the 3D magnetic correlation length $\xi_{3 \mathrm{D}}$ and $Q_{c}$ is the length [Q $=(4 \pi / \lambda) \sin \theta]$ of the scattering vector at which the peak of $I(Q)$ occurs. The 2D correlation length $\xi_{2 \mathrm{D}}$, obtained from the analysis using the Warren function, was found to be 30 , 50 , and $70 \AA$ at 180,175 , and $170 \mathrm{~K}$, respectively. It is evident that the Lorentzian function gives a better representation of data. $\xi_{3 \mathrm{D}}$ was found to be $8.4 \pm 1.9,8.7 \pm 1.3$, and $8.8 \pm 0.9 \AA$ at 180,175 , and $170 \mathrm{~K}$, respectively and the corresponding $Q_{c}$ values are $1.16 \pm 0.01,1.162 \pm 0.008$, and $1.168 \pm 0.006 \AA^{-1}$, respectively. The broad diffuse peak over $170-180 \mathrm{~K}$, therefore, indicates the presence of 3D shortrange spin-spin correlation between Mn spins. The broad peaks at $T=180-170 \mathrm{~K}$ and saw-tooth type asymmetric Bragg peaks at $T=165-160 \mathrm{~K}$ are observed around the $Q$ 


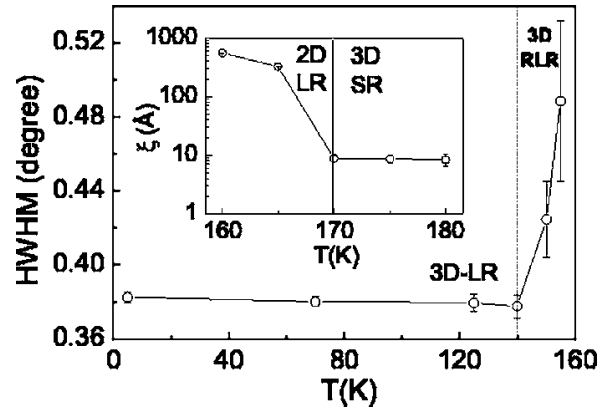

FIG. 4. Temperature dependence of HWHM, averaged for the 3D Bragg peaks (100) and (001). The inset shows the temperature variation of the spin-spin correlation length $\xi$. Various magnetic ordering regions are indicated. 3D-RLR region corresponds to 3D long range order with restricted (reduced) correlation length along [010].

values where the (100) and (001) 3D antiferromagnetic Bragg peaks appear. This suggests that the Mn moments in the 2D structure are arranged in the same way as the moments in the 3D antiferromagnetic structure. Based on this, the asymmetric saw-tooth type Bragg peaks, observed at $2 \theta=51.30$ and $52.70 \mathrm{deg}$, are indexed as $(h=1, l=0)$ and $(h=0, l=1)$, respectively.

Since the bilayers of $\mathrm{MnO}_{6}$ octahedra are separated by single layers of $\mathrm{GaO}_{4}$ tetrahedra along [010], the superexchange interactions are suppressed perpendicular to the $\mathrm{MnO}_{6}$ octahedral layers due to the presence of the filled electron orbitals of $\mathrm{Ga}\left(d^{10}\right)$ ions. It is therefore, expected (as dictated by crystallography) that $J_{a c} \gg J_{b}{ }^{14,15,20}$ We therefore, expect to observe magnetic correlations between $\mathrm{Mn}$ spins within the $a-c$ plane. The observation of 2D LRO over a relatively wide temperature range $(165-160 \mathrm{~K})$ indicates that along the $c$ direction some hindering process to the 3D LRO is present which could be due to the microstructure of the sample. For example the presence of stacking disorder between the $\mathrm{MnO}_{6}$ sheets due to defects or other inhomogeneities can strongly confine (pin) the 2D correlation. In fact, electron microscopy study on the present compound suggests that the local microstructure is more complex than the average structure (space group $P c m 2_{1}$ ) revealed by neutron diffraction. ${ }^{15}$ The observation of Ibm2-type atomic arrangements in the $P \mathrm{~cm} 2_{1}$-type matrix was interpreted as a disorder phenomenon at the level of the $[\mathrm{GaO}]$ layers. ${ }^{15}$ In the present study the effect of hindering process is found to continue even at lower $T$ showing the restricted 3D correlation (asymmetric broad 3D Bragg peaks) over $\sim 150-155 \mathrm{~K}$. Further measurements on single crystals with varying disorder would be beneficial to shed more light in this respect.

In Fig. 4 we present a tentative phase diagram of the studied compound. From the temperature dependence of the magnetic patterns and the derived $\xi(T)$ values, the presence of SR-3D and LR-2D antiferromagnetic correlations in the $a-c$ plane are found at $170 \leq T \leq 180 \mathrm{~K}$ and $155<T$ $<170 \mathrm{~K}$, respectively. By studying the half width at half maximum (HWHM) of the (100) and (001) Bragg peaks we found the presence of LR-3D antiferromagnetic ordering at $T \leq 140 \mathrm{~K}$ where the Bragg peak widths are limited by the instrumental resolution, whereas, for $150 \leq T \leq 155 \mathrm{~K}$, the 3D Bragg peak width starts to increase and the peak shapes start becoming asymmetric indicating that magnetic correlations along [010], between LR-2D-ordered manganite bilayers, start to decrease.

The 3D SR $\rightarrow$ 2D LR $\rightarrow$ 3D LR magnetic ordering is accompanied by a tenfold decrease of resistivity and a large negative $\mathrm{MR}(\sim 50 \%) .{ }^{14}$ Here the $2 \mathrm{D}$ (in-plane $\mathrm{MnO}_{6}$ layer) ordering is antiferromagnetic whereas, at $T \leq T_{\mathrm{N}-3 \mathrm{D}}$ a ferromagnetic interlayer coupling is observed. Over the $2 \mathrm{D}$ magnetic ordering temperature region, charge carriers are confined within the $\mathrm{MnO}_{6}$ layers. With the onset of 3D LRO, charge carriers have also access to the third dimension ( $b$ axis) along which a ferromagnetic exchange between spins exists. This results a sharp fall of resistivity with 3D Néel ordering. Large negative MR effect is closely related to the change in the interbilayer ferromagnetic coupling as found in other layered systems such as $\mathrm{La}_{3-x} \mathrm{Sr}_{x} \mathrm{Mn}_{2} \mathrm{O}_{7}{ }^{8}$

\section{CONCLUSION}

The present study has shown clear crossovers of SR 3D $\rightarrow$ LR $2 \mathrm{D} \rightarrow$ LR 3D magnetic order for a system with quite high LRO temperatures $\left(T_{\mathrm{N}-2 \mathrm{D}} \sim 165 \mathrm{~K}\right.$ and $\left.T_{\mathrm{N}-3 \mathrm{D}} \sim 150 \mathrm{~K}\right)$. The observations of a marked decrease in resistivity and a high MR at $T \sim T_{\mathrm{N}-3 \mathrm{D}}$ suggest that the electronic and magnetic properties are strongly coupled and dimensional dependent. Here the dimensionality crossover phenomenon of electronic and magnetic properties is found in a system where $3 d(\mathrm{Mn})$ magnetism, and superexchange interactions are involved. These findings should stimulate a motivation for studying 2D magnetic ordering in other layered materials including the important $A_{n+1} \mathrm{Mn}_{n} \mathrm{O}_{3 n+1}$ Ruddlesden-Popper (RP) series and the interplay between their anisotropic magnetic, transport and structural properties from the CMR and dimensionality crossover point of view. We believe that the results would be very useful to theoreticians working on low dimensional systems.

\section{ACKNOWLEDGMENTS}

The authors are grateful to P. D. Battle and M. J. Rosseinsky for providing us the sample and fruitful discussions. Financial supports by the European Commission through the Research Training Network "SCOOTMO" Contract No. HPRN-CT-2002-00293, the Spanish Ministry of Science (through projects SAB2003-0255, MAT2002-04657 and MAT2005-05565-C02-02) and the NMI3_CT_2003-505925 project are acknowledged. 
*Corresponding author. Electronic address: smyusuf@barc.gov.in

${ }^{1}$ N. D. Mermin and H. Wagner, Phys. Rev. Lett. 17, 1133 (1966).

${ }^{2}$ P. Bruno, Phys. Rev. Lett. 87, 137203 (2001).

${ }^{3}$ A. S. T. Pires, Phys. Rev. B 53, 5123 (1996).

${ }^{4}$ J. R. de Sousa and N. S. Branco, Phys. Rev. B 72, 134421 (3005).

${ }^{5}$ A. Grechnev, V. Yu. Irkhin, M. I. Katsnelson, and O. Eriksson, Phys. Rev. B 71, 024427 (2005).

${ }^{6}$ N. Majlis, S. Selzer, and G. C. Strinati, Phys. Rev. B 45, 7872 (1992); N. Majlis, S. Selzer, and G. C. Strinati, ibid. 48, 957 (1993).

${ }^{7}$ J. van Duijn, J. P. Attfield, R. Watanuki, K. Suzuki, and R. K. Heenan, Phys. Rev. Lett. 90, 087201 (2003).

${ }^{8}$ T. Kimura and Y. Tokura, Annu. Rev. Mater. Sci. 30, 451 (2000).

${ }^{9}$ J. W. Lynn, T. W. Clinton, W.-H. Li, R. W. Erwin, J. Z. Liu, K. Vandervoort, and R. N. Shelton, Phys. Rev. Lett. 63, 2606 (1989).

${ }^{10}$ H. Zhang, J. W. Lynn, and D. E. Morris, Phys. Rev. B 45, 10022 (1992).

${ }^{11}$ S. Y. Wu, W.-H. Li, K. C. Lee, J. W. Lynn, T. H. Meen, and H. D. Yang, Phys. Rev. B 54, 10019 (1996).

${ }^{12}$ R. A. Cowley, M. Hagen, and D. P. Belanger, J. Phys. C 17, 3763 (1984).

${ }^{13}$ R. J. Birgeneau, J. Skalyo, and G. Shirane, Phys. Rev. B 3, 1736
(1971).

${ }^{14}$ P. D Battle, S. J. Blundell, P. N. Santhosh, M. J. Rosseinsky, and C. Steer, J. Phys.: Condens. Matter 14, 13569 (2002).

${ }^{15}$ P. D. Battle, S. J. Blundell, M. L. Brooks, M. Hervieu, C. Kapusta, T. Lancaster, S. P. Nair, C. J. Oates, F. L. Pratt, M. J. Rosseinsky, R. Ruiz-Bustos, M. Sikora, and C. A. Steer, J. Am. Chem. Soc. 126, 12517 (2004).

${ }^{16}$ A. P. Ramirez, J. Phys.: Condens. Matter 9, 8171 (1997).

${ }^{17}$ T. Roisnel and J. Rodriguez-Carvajal, WinPLOTR: a Windows tool for powder diffraction patterns analysis, Materials Science Forum, Proceedings of the Seventh European Powder Diffraction Conference (EPDIC 7), 2000, p. 118-123, edited by R. Delhez and E. J. Mittenmeijer; J. Rodriguez-Carvajal and T. Roisnel, FullProf.98 and WinPLOTR: New Windows 95/NT Applications for Diffraction Commission For Powder Diffraction, International Union for Crystallography, Newsletter N_20 (MayAugust) Summer 1998.

${ }^{18}$ M. Bander and D. L. Mills, Phys. Rev. B 38, 12015 (1988).

${ }^{19}$ B. E. Warren, Phys. Rev. 59, 693 (1941).

${ }^{20}$ S. J. Blundell, J. A. Stride, M. L. Brooks, C. A. Steer, S. P. Nair, P. D Battle, and M. J. Rosseinsky, J. Phys.: Condens. Matter 17, 99 (2005). 